\title{
Tobacco Product Use Among Adults — United States, 2019
}

\author{
Monica E. Cornelius, $\mathrm{PhD}^{1}$; Teresa W. Wang, $\mathrm{PhD}^{1}$; Ahmed Jamal, MBBS ${ }^{1}$; Caitlin G. Loretan, MPH${ }^{1}$; Linda J. Neff, PhD ${ }^{1}$
}

Cigarette smoking remains the leading cause of preventable disease and death in the United States (1). The prevalence of current cigarette smoking among U.S. adults has declined over the past several decades, with a prevalence of $13.7 \%$ in 2018 (2). However, a variety of combustible, noncombustible, and electronic tobacco products are available in the United States $(1,3)$. To assess recent national estimates of tobacco product use among U.S. adults aged $\geq 18$ years, CDC analyzed data from the 2019 National Health Interview Survey (NHIS). In 2019, an estimated 50.6 million U.S. adults (20.8\%) reported currently using any tobacco product, including cigarettes $(14.0 \%)$, e-cigarettes $(4.5 \%)$, cigars $(3.6 \%)$, smokeless tobacco $(2.4 \%)$, and pipes* $(1.0 \%){ }^{\dagger}$ Most current tobacco product users $(80.5 \%)$ reported using combustible products (cigarettes, cigars, or pipes), and $18.6 \%$ reported using two or more tobacco products. ${ }^{\S}$ The prevalence of any current tobacco product use was higher among males; adults aged $\leq 65$ years; non-Hispanic American Indian/ Alaska Native (AI/AN) adults; those whose highest level of educational attainment was a General Educational Development (GED) certificate; those with an annual household income $<\$ 35,000$; lesbian, gay, or bisexual (LGB) adults; uninsured adults and those with Medicaid; those with a disability; or those with mild, moderate, or severe generalized anxiety disorder. E-cigarette use was highest among adults aged 18-24 years $(9.3 \%)$, with over half $(56.0 \%)$ of these young adults reporting that they had never smoked cigarettes. Implementing comprehensive, evidence-based, population level interventions (e.g., tobacco price increases, comprehensive smoke-free policies, highimpact antitobacco media campaigns, and barrier-free cessation coverage), in coordination with regulation of the manufacturing, marketing, and sale of all tobacco products, can reduce tobaccorelated disease and death in the United States $(1,4)$. As part of a comprehensive approach, targeted interventions are also warranted to reach subpopulations with the highest prevalence of use, which might vary by tobacco product type.

NHIS is an annual, nationally representative, household survey of the noninstitutionalized U.S. civilian population. The

\footnotetext{
*The use of regular pipe, water pipe, or hookah was assessed together using a single question. Interviewers could read the following sentences, if necessary: "A hookah is a type of water pipe. It is sometimes called a narghile pipe. Do not include electronic hookahs or e-hookahs." "Do not include electronic pipes or e-pipes. Do not include pipes filled with substances other than tobacco." $\dagger$ Categories are not mutually exclusive.

$\$$ Current use of two or more tobacco products was defined as "every day" or "some day" use of two or more of the following tobacco products: cigarettes ( $\geq 100$ cigarettes during lifetime); cigars, cigarillos, or filtered little cigars; pipes, water pipes, or hookahs; electronic cigarettes; or smokeless tobacco products. https://www.cdc.gov/nchs/nhis/data-questionnaires-documentation.htm.
}

2019 NHIS Sample Adult component included 31,997 adults aged $\geq 18$ years; the response rate was $59.1 \%$ (5). Data were weighted to account for complex survey design and provide nationally representative estimates. Use of five tobacco product types was assessed: cigarettes, cigars (cigars, cigarillos, or filtered little cigars), pipes (regular pipes, water pipes, or hookahs), e-cigarettes, and smokeless tobacco (chewing tobacco, snuff, dip, snus, or dissolvable tobacco). Current cigarette smokers reported having smoked $\geq 100$ cigarettes during their lifetime and reported that they smoked "every day" or "some days" at the time of survey. Current users of all other tobacco products reported using these products "every day" or "some days" at the time of survey. Prevalence estimates for current use of each tobacco product type, any tobacco product, any combustible tobacco product, and two or more tobacco products were calculated. Estimates were calculated overall and by sex, age, race/ethnicity, U.S. Census region, ${ }^{* *}$ education (adults aged $\geq 25$ years), marital status, annual household income, ${ }^{\dagger \dagger}$ sexual orientation, ${ }^{\mathbb{\$}}$ health insurance coverage, ${ }^{\mathbf{9}}$ disability status, ${ }^{* * *}$ and indication of generalized anxiety disorder (GAD-7) ${ }^{\dagger \dagger \dagger}$ The distribution of age groups was assessed among current users of each tobacco product, any tobacco product,

\footnotetext{
** Northeast: Connecticut, Maine, Massachusetts, New Hampshire, New Jersey, New York, Pennsylvania, Rhode Island, and Vermont. Midwest: Illinois, Indiana, Iowa, Kansas, Michigan, Minnesota, Missouri, Nebraska, North Dakota, Ohio, South Dakota, and Wisconsin. South: Alabama, Arkansas, Delaware, District of Columbia, Florida, Georgia, Kentucky, Louisiana, Maryland, Mississippi, North Carolina, Oklahoma, South Carolina, Tennessee, Texas, Virginia, and West Virginia. West: Alaska, Arizona, California, Colorado, Hawaii, Idaho, Montana, Nevada, New Mexico, Oregon, Utah, Washington, and Wyoming.

$\dagger \dagger$ Based on the imputed sample adult family income (grouped) variable $(n=31,997)$. $\mathrm{ftp}: / /$ ftp.cdc.gov/pub/Health_Statistics/NCHS/Dataset_Documentation/ NHIS/2019/srvydesc-508.pdf.

$\$ \$$ Sexual orientation was determined using the question "Which of the following best represents how you think of yourself?" Response options included "gay," "straight, that is, not gay," "bisexual," "something else," and "I don't know the answer" among male respondents, and "lesbian or gay," "straight, that is, not lesbian or gay," "bisexual, "something else," and "I don't know the answer" among female respondents. Respondents were considered to be lesbian, gay, or bisexual if they responded "gay," "lesbian or gay," or "bisexual."

IS Private coverage: includes adults who had any comprehensive private insurance plan (including health maintenance organizations and preferred provider organizations). Medicaid: for adults aged $<65$ years, includes those who did not have private coverage, but who had Medicaid or other state-sponsored health plans, including Children's Health Insurance Program (CHIP). For adults aged $\geq 65$ years, includes adults aged $\geq 65$ years who did not have any private coverage but had Medicare and Medicaid or other state-sponsored health plans; Medicare only: includes adults aged $\geq 65$ years who only had Medicare coverage; Other coverage: includes adults who did not have private insurance, Medicaid, or other public coverage, but who had any type of military coverage, coverage from other government programs, or Medicare. Uninsured: includes adults who did not indicate that they were covered at the time of the interview under private health insurance, Medicare, Medicaid, CHIP, a state-sponsored health plan, other government programs, or military coverage.
} 
combustible products, and two or more tobacco products. Among e-cigarette users, the percentage of current, ${ }^{\$ \$ \$}$ former, ${ }^{99}$ and never**** cigarette smokers was assessed by age group. SAS-callable SUDAAN software (version 11.0.3; RTI International) was used to conduct all analyses.

Among U.S. adults in 2019, 20.8\% (estimated 50.6 million) currently used any tobacco product, $16.7 \%$ ( 40.8 million) used any combustible tobacco product, and 3.9\% (9.4 million) used two or more tobacco products (Table). Cigarettes were the most commonly used tobacco product (14.0\%; 34.1 million). Prevalence of use of other tobacco products was as follows: e-cigarettes (4.5\%; 10.9 million); cigars (3.6\%; 8.7 million); smokeless tobacco $(2.4 \% ; 5.9$ million); and pipes $(1.0 \%$; 2.4 million). Combustible tobacco products were used by $80.5 \%$ of current tobacco product users. Use of two or more tobacco products was reported by $18.6 \%$ of current tobacco product users.

The tobacco product with the highest percentage of users aged 18-24 (24.5\%) and 25-44 years (49.3\%) was e-cigarettes (Figure 1). The tobacco product with the highest percentage of users aged $45-64(40.2 \%)$ and $\geq 65$ years $(12.3 \%)$ was cigarettes. Among current e-cigarette users, $36.9 \%$ were current cigarette smokers, $39.5 \%$ were former cigarette smokers, and $23.6 \%$ were never cigarette smokers (Figure 2). The percentage of e-cigarette users who were never smokers was highest

${ }^{* * *}$ Disability was defined based on self-reported presence of selected limitations including vision, hearing, mobility, remembering, self-care, communication. Respondents had to answer "A lot of difficulty" or "Cannot do at all/unable to do" to one of the following questions: "Do you have difficulty seeing, even when wearing glasses?," Do you have difficulty hearing, even when using a hearing aid?," "Do you have any difficulty walking or climbing steps?," "Using your usual language, do you have difficulty communicating, for example, understanding or being understood?," "Do you have difficulty remembering or concentrating?," "Do you have difficulty with self-care, such as washing all over or dressing?" to be coded as having a disability; those who responded "no difficulty" or "some difficulty" to all six questions were coded as not having a disability. These six questions are based on the short set of questions recommended by the Washington Group on Disability Statistics (https://www.cdc.gov/nchs/washington_group/index.htm).

ti† Based on the 7-item Generalized Anxiety Disorder scale (GAD-7) recode of none/minimal (values 0-4), mild (values 5-9), moderate (values 10-14) and severe (values 15-21). Adults were asked how often they have been bothered by the following symptoms in the past 2 weeks: "Feeling nervous, anxious, or on edge"; "Not being able to stop or control worrying"; "Worrying too much about different things"; "Trouble relaxing"; "Being so restless that it's hard to sit still"; "Becoming easily annoyed or irritable"; and "Feeling afraid as if something awful might happen." Response options were "not at all," "several days," "more than half the days," and "nearly every day," scored as 0 to 3 points, respectively, and then summed into a total score.

$\$ \$ \$$ Current cigarette smokers were defined as adults who reported smoking $\geq 100$ cigarettes during their lifetime and smoked cigarettes "every day" or "some days" at the time of the interview (only other response options were "not at all, refused, and don't know").

999 Former cigarette smokers were defined as adults who had smoked $\geq 100$ cigarettes in their lifetime but reported smoking "not at all" at the time of the interview.

**** Never cigarette smokers were defined as adults who had not smoked $\geq 100$ cigarettes in their lifetime.
(56.0\%) among the 18-24 age group and decreased with increasing age. The percentage of e-cigarette users who were former smokers was lowest $(20.5 \%)$ among the 18-24 age group and increased with increasing age. Many adults in all age groups were dual users of e-cigarettes and cigarettes.

The prevalence of any current tobacco product use was higher among males (26.2\%) than among females (15.7\%) and among those aged $25-44$ years (25.3\%), 45-64 years (23.0\%), or $18-24$ years $(18.2 \%)$ than among those aged $\geq 65$ years (11.4\%) (Table). Current tobacco product use was also higher among non-Hispanic AI/AN adults (29.3\%), non-Hispanic adults of other ${ }^{\dagger \dagger \dagger}$ races $(28.1 \%)$, non-Hispanic White adults (23.3\%), non-Hispanic Black adults (20.7\%), and Hispanic or Latino adults (13.2\%) than among non-Hispanic Asian adults (11.0\%); and among those living in the Midwest $(23.7 \%)$ or South $(22.9 \%)$ than among those in the Northeast $(18.5 \%)$ or West $(16.4 \%)$. The prevalence of current tobacco product use was higher among those whose highest educational attainment was a GED (43.7\%) than among those with other levels of education; among those who were divorced/separated/ widowed $(23.5 \%)$ or single/never married/not living with a partner $(23.0 \%)$ than among those married/living with a partner (19.2\%); among those who had annual household income of $<\$ 35,000(27.0 \%)$ than among those with higher income; and among LGB adults (29.9\%) than among those who were heterosexual/straight (20.5\%). Prevalence was also higher among adults who were uninsured (30.2\%), insured by Medicaid $(30.0 \%)$, or had some other public insurance $(25.6 \%)$ than among those with private insurance (18.0\%) or Medicare only (11.4\%); among those who had a disability (26.9\%) compared with those without $(20.1 \%)$; and among those who had GAD-7 scores indicating mild (30.4\%), moderate $(34.2 \%)$ or severe $(45.3 \%)$ anxiety than among those indicating no or minimal (18.4\%) anxiety.

\section{Discussion}

In 2019, approximately one in five U.S. adults (50.6 million) reported currently using any tobacco product. Cigarettes were the most commonly used tobacco product among adults, and combustible tobacco products (cigarettes, cigars, or pipes) were used by most $(80.5 \%)$ adult tobacco product users. Most of the death and disease from tobacco use in the United States is

††† The following four non-Hispanic single race categories were available for sample adults in the 2019 NHIS public use files: 1) White; 2) Black or African American; 3) Asian; and 4) American Indian or Alaska Native (AI/AN). Exclusive from these groups, the "non-Hispanic, Other" category in this report includes those adults who were categorized as "non-Hispanic AI/AN and any other group" or "other single and multiple races." The only multiracial category available was "non-Hispanic AI/AN and any other group." ftp://ftp.cdc.gov/pub/Health_Statistics/NCHS/Dataset_ Documentation/NHIS/2019/srvydesc-508.pdf. 
TABLE. Percentage of adults aged $\geq 18$ years who reported tobacco product use "every day" or "some days," by tobacco product and selected characteristics - National Health Interview Survey, United States, 2019

\begin{tabular}{|c|c|c|c|c|c|c|c|c|}
\hline \multirow[b]{2}{*}{ Characteristic } & \multicolumn{8}{|c|}{$\%(95 \% \mathrm{Cl})$} \\
\hline & $\begin{array}{l}\text { Any tobacco } \\
\text { product }^{*}\end{array}$ & $\begin{array}{l}\text { Any combustible } \\
\text { product }^{\dagger}\end{array}$ & Cigarettes ${ }^{\S}$ & $\begin{array}{c}\text { Cigars/Cigarillos/ } \\
\text { Filtered } \\
\text { little cigars }\end{array}$ & $\begin{array}{l}\text { Regular pipe/ } \\
\text { Water pipe/ } \\
\text { Hookah** }\end{array}$ & E-cigarettes ${ }^{\dagger+}$ & $\begin{array}{l}\text { Smokeless } \\
\text { tobacco }^{\S \S}\end{array}$ & $\begin{array}{l}\geq 2 \text { Tobacco } \\
\text { products }\end{array}$ \\
\hline Overall & $20.8(20.2-21.4)$ & $16.7(16.1-17.3)$ & $14.0(13.5-14.5)$ & $3.6(3.3-3.9)$ & $1.0(0.9-1.1)$ & $4.5(4.2-4.8)$ & $2.4(2.2-2.6)$ & $3.9(3.6-4.2)$ \\
\hline \multicolumn{9}{|l|}{ Sex } \\
\hline Male & $26.2(25.3-27.1)$ & $20.1(19.3-20.9)$ & $15.3(14.5-16.1)$ & $6.3(5.8-6.8)$ & $1.5(1.3-1.7)$ & $5.5(5.0-6.0)$ & $4.7(4.2-5.2)$ & $5.7(5.2-6.2)$ \\
\hline Female & $15.7(14.9-16.5)$ & $13.6(12.9-14.3)$ & $12.7(12.0-13.4)$ & $1.1(0.9-1.3)$ & $0.5(0.4-0.6)$ & $3.5(3.1-3.9)$ & $0.3(0.2-0.4)$ & $2.2(1.9-2.5)$ \\
\hline \multicolumn{9}{|l|}{ Age group (yrs) } \\
\hline $18-24$ & $18.2(16.2-20.2)$ & $11.2(9.7-12.7)$ & $8.0(6.7-9.3)$ & $3.8(2.8-4.8)$ & $1.7(1.1-2.3)$ & $9.3(7.9-10.7)$ & $2.2(1.4-3.0)$ & $5.2(4.1-6.3)$ \\
\hline $25-44$ & $25.3(24.2-26.4)$ & $20.1(19.1-21.1)$ & $16.7(15.8-17.6)$ & $4.4(3.9-4.9)$ & $1.3(1.0-1.6)$ & $6.4(5.8-7.0)$ & $3.2(2.8-3.6)$ & $5.5(4.9-6.1)$ \\
\hline $45-64$ & $23.0(21.9-24.1)$ & $19.5(18.5-20.5)$ & $17.0(16.0-18.0)$ & $3.7(3.3-4.1)$ & $0.6(0.4-0.8)$ & $3.0(2.6-3.4)$ & $2.5(2.1-2.9)$ & $3.4(3.0-3.8)$ \\
\hline$\geq 65$ & $11.4(10.6-12.2)$ & $9.9(9.2-10.6)$ & $8.2(7.5-8.9)$ & $2.0(1.6-2.4)$ & $0.5(0.3-0.7)$ & $0.8(0.6-1.0)$ & $1.2(0.9-1.5)$ & $1.3(1.0-1.6)$ \\
\hline \multicolumn{9}{|l|}{ Race/Ethnicity*** } \\
\hline White, non-Hispanic & $23.3(22.5-24.1)$ & $18.3(17.6-19.0)$ & $15.5(14.8-16.2)$ & $3.8(3.5-4.1)$ & $1.0(0.8-1.2)$ & $5.1(4.7-5.5)$ & $3.4(3.1-3.7)$ & $4.5(4.1-4.9)$ \\
\hline Black, non-Hispanic & $20.7(19.0-22.4)$ & $18.6(17.0-20.2)$ & $14.9(13.4-16.4)$ & $4.4(3.5-5.3)$ & $1.1(0.7-1.5)$ & $3.4(2.6-4.2)$ & $0.5(0.3-0.7)$ & $3.3(2.5-4.1)$ \\
\hline Asian, non-Hispanic & $11.0(9.0-13.0)$ & $8.6(6.7-10.5)$ & $7.2(5.4-9.0)$ & $1.2(0.6-1.8)$ & - +t+ & $2.7(1.7-3.7)$ & - & $1.4(0.8-2.0)$ \\
\hline $\begin{array}{c}\text { American Indian/Alaska } \\
\text { Native, non-Hispanic }\end{array}$ & $29.3(16.4-42.2)$ & $22.3(10.5-34.1)$ & $20.9(9.9-31.9)$ & - & - & - & - & - \\
\hline Hispanic & $13.2(11.9-14.5)$ & $11.2(10.0-12.4)$ & $8.8(7.8-9.8)$ & $3.0(2.3-3.7)$ & $0.8(0.5-1.1)$ & $2.8(2.2-3.4)$ & $0.5(0.3-0.7)$ & $2.2(1.7-2.7)$ \\
\hline Other, non-Hispanic & $28.1(23.4-32.8)$ & $22.0(17.7-26.3)$ & $19.7(15.7-23.7)$ & $3.1(1.6-4.6)$ & - & $9.3(6.0-12.6)$ & - & $7.5(4.7-10.3)$ \\
\hline \multicolumn{9}{|l|}{ U.S. Census region $§ \S \S$} \\
\hline Northeast & $18.5(17.1-19.9)$ & $16.0(14.7-17.3)$ & $12.8(11.5-14.1)$ & $3.8(3.1-4.5)$ & $0.8(0.5-1.1)$ & $3.3(2.7-3.9)$ & $1.1(0.7-1.5)$ & $2.9(2.4-3.4)$ \\
\hline Midwest & $23.7(22.2-25.2)$ & $19.1(17.8-20.4)$ & $16.4(15.2-17.6)$ & $3.9(3.2-4.6)$ & $1.0(0.7-1.3)$ & $4.5(3.9-5.1)$ & $3.1(2.5-3.7)$ & $4.1(3.5-4.7)$ \\
\hline South & $22.9(21.8-24.0)$ & $18.2(17.2-19.2)$ & $15.4(14.5-16.3)$ & $3.9(3.4-4.4)$ & $1.0(0.8-1.2)$ & $4.9(4.3-5.5)$ & $3.0(2.6-3.4)$ & $4.5(4.0-5.0)$ \\
\hline West & $16.4(15.3-17.5)$ & $12.6(11.6-13.6)$ & $10.4(9.4-11.4)$ & $2.6(2.2-3.0)$ & $1.0(0.7-1.3)$ & $4.4(3.8-5.0)$ & $1.9(1.4-2.4)$ & $3.4(2.9-3.9)$ \\
\hline \multicolumn{9}{|c|}{ Education (adults aged $\geq 25$ years) } \\
\hline $\begin{array}{l}\text { 0-12 years } \\
\text { (no diploma) }\end{array}$ & $26.4(24.2-28.6)$ & $23.5(21.4-25.6)$ & $21.6(19.5-23.7)$ & $3.0(2.1-3.9)$ & $1.2(0.6-1.8)$ & $3.0(2.2-3.8)$ & $2.9(2.1-3.7)$ & $4.0(3.1-4.9)$ \\
\hline $\begin{array}{l}\text { General Educational } \\
\text { Development }\end{array}$ & $43.7(39.1-48.3)$ & $37.1(32.8-41.4)$ & $35.3(31.1-39.5)$ & $5.2(3.2-7.2)$ & - & $7.8(5.5-10.1)$ & $4.9(2.6-7.2)$ & $8.9(6.4-11.4)$ \\
\hline High school diploma & $26.4(25.0-27.8)$ & $21.9(20.6-23.2)$ & $19.6(18.3-20.9)$ & $3.7(3.1-4.3)$ & $0.8(0.6-1.0)$ & $4.3(3.7-4.9)$ & $3.5(2.9-4.1)$ & $4.8(4.1-5.5)$ \\
\hline $\begin{array}{l}\text { Some college, } \\
\text { no diploma }\end{array}$ & $24.8(23.2-26.4)$ & $20.6(19.1-22.1)$ & $17.7(16.3-19.1)$ & $3.7(2.9-4.5)$ & $0.9(0.6-1.2)$ & $5.0(4.2-5.8)$ & $2.0(1.5-2.5)$ & $3.9(3.2-4.6)$ \\
\hline $\begin{array}{l}\text { Associate degree } \\
\text { (academic or technical/ } \\
\text { vocational) }\end{array}$ & $21.2(19.6-22.8)$ & $16.8(15.4-18.2)$ & $14.0(12.7-15.3)$ & $3.8(3.1-4.5)$ & $0.7(0.3-1.1)$ & $4.5(3.7-5.3)$ & $2.8(2.2-3.4)$ & $4.0(3.3-4.7)$ \\
\hline $\begin{array}{l}\text { Undergraduate degree } \\
\text { (bachelor's) }\end{array}$ & $13.1(12.2-14.0)$ & $10.0(9.1-10.9)$ & $6.9(6.2-7.6)$ & $3.4(2.8-4.0)$ & $0.9(0.6-1.2)$ & $3.2(2.7-3.7)$ & $1.5(1.2-1.8)$ & $2.4(2.0-2.8)$ \\
\hline $\begin{array}{l}\text { Graduate degree } \\
\text { (master's, professional, } \\
\text { or doctoral) }\end{array}$ & $8.7(7.8-9.6)$ & $7.1(6.2-8.0)$ & $4.0(3.3-4.7)$ & $3.2(2.6-3.8)$ & $0.7(0.4-1.0)$ & $1.5(1.1-1.9)$ & $1.0(0.7-1.3)$ & $1.5(1.1-1.9)$ \\
\hline \multicolumn{9}{|l|}{ Marital status } \\
\hline $\begin{array}{l}\text { Married/Living with } \\
\text { partner }\end{array}$ & $19.2(18.5-19.9)$ & $15.3(14.6-16.0)$ & $12.4(11.8-13.0)$ & $3.5(3.1-3.9)$ & $0.8(0.6-1.0)$ & $3.9(3.5-4.3)$ & $2.5(2.2-2.8)$ & $3.2(2.9-3.5)$ \\
\hline $\begin{array}{l}\text { Divorced/Separated/ } \\
\text { Widowed }\end{array}$ & $23.5(22.2-24.8)$ & $20.6(19.4-21.8)$ & $19.0(17.9-20.1)$ & $3.0(2.5-3.5)$ & $0.8(0.5-1.1)$ & $3.3(2.8-3.8)$ & $2.1(1.7-2.5)$ & $4.2(3.6-4.8)$ \\
\hline $\begin{array}{l}\text { Single/Never married/ } \\
\text { Not living with } \\
\text { a partner }\end{array}$ & $23.0(21.6-24.4)$ & $17.8(16.5-19.1)$ & $14.6(13.4-15.8)$ & $4.1(3.5-4.7)$ & $1.7(1.3-2.1)$ & $6.9(6.1-7.7)$ & $2.5(1.9-3.1)$ & $5.3(4.6-6.0)$ \\
\hline \multicolumn{9}{|c|}{ 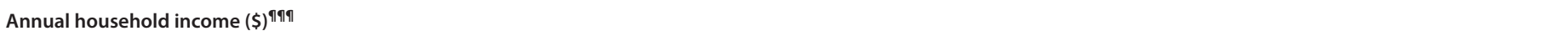 } \\
\hline$<35,000$ & $27.0(25.7-28.3)$ & $23.2(22.0-24.4)$ & $21.4(20.2-22.6)$ & $3.2(2.8-3.6)$ & $1.2(0.9-1.5)$ & $5.0(4.4-5.6)$ & $2.0(1.6-2.4)$ & $4.8(4.2-5.4)$ \\
\hline $35,000-74,999$ & $22.0(20.9-23.1)$ & $18.1(17.1-19.1)$ & $15.7(14.7-16.7)$ & $3.2(2.7-3.7)$ & $1.1(0.8-1.4)$ & $4.5(4.0-5.0)$ & $2.5(2.1-2.9)$ & $4.3(3.8-4.8)$ \\
\hline $75,000-99,999$ & $18.8(17.3-20.3)$ & $14.5(13.1-15.9)$ & $11.4(10.1-12.7)$ & $3.9(3.1-4.7)$ & $1.1(0.6-1.6)$ & $4.6(3.7-5.5)$ & $2.4(1.8-3.0)$ & $3.5(2.7-4.3)$ \\
\hline$\geq 100,000$ & $15.1(14.1-16.1)$ & $10.8(10.0-11.6)$ & $7.1(6.4-7.8)$ & $4.1(3.6-4.6)$ & $0.7(0.5-0.9)$ & $3.8(3.3-4.3)$ & $2.7(2.2-3.2)$ & $2.8(2.4-3.2)$ \\
\hline \multicolumn{9}{|l|}{ Sexual orientation } \\
\hline Heterosexual/Straight & $20.5(19.9-21.1)$ & $16.5(15.9-17.1)$ & $13.8(13.2-14.4)$ & $3.6(3.3-3.9)$ & $0.9(0.8-1.0)$ & $4.2(3.9-4.5)$ & $2.5(2.3-2.7)$ & $3.8(3.5-4.1)$ \\
\hline Lesbian, Gay, or Bisexual & $29.9(25.9-33.9)$ & $22.7(19.2-26.2)$ & $19.2(16.1-22.3)$ & $4.7(2.9-6.5)$ & $2.3(1.1-3.5)$ & $11.5(8.7-14.3)$ & - & $6.9(5.0-8.8)$ \\
\hline \multicolumn{9}{|c|}{ Health insurance coverage $\mathrm{e}^{* * * *}$} \\
\hline Private insurance & $18.0(17.3-18.7)$ & $13.7(13.1-14.3)$ & $10.7(10.1-11.3)$ & $3.6(3.3-3.9)$ & $0.9(0.7-1.1)$ & $4.3(3.9-4.7)$ & $2.5(2.2-2.8)$ & $3.3(3.0-3.6)$ \\
\hline Medicaid & $30.0(27.9-32.1)$ & $26.8(24.8-28.8)$ & $24.9(22.9-26.9)$ & $3.3(2.6-4.0)$ & $1.1(0.7-1.5)$ & $5.0(4.0-6.0)$ & $1.8(1.3-2.3)$ & $5.3(4.3-6.3)$ \\
\hline $\begin{array}{l}\text { Medicare only } \\
\text { (aged } \geq 65 \text { yrs) }\end{array}$ & $11.4(9.9-12.9)$ & $10.1(8.7-11.5)$ & $8.6(7.3-9.9)$ & $1.8(1.2-2.4)$ & - & $1.0(0.6-1.4)$ & - & $1.2(0.7-1.7)$ \\
\hline Other public insurance & $25.6(23.2-28.0)$ & $20.8(18.7-22.9)$ & $17.8(15.9-19.7)$ & $5.4(3.9-6.9)$ & $1.1(0.6-1.6)$ & $4.4(3.2-5.6)$ & $3.4(2.2-4.6)$ & $5.2(4.0-6.4)$ \\
\hline Uninsured & $30.2(28.0-32.4)$ & $24.9(22.9-26.9)$ & $22.5(20.6-24.4)$ & $4.1(3.1-5.1)$ & $1.3(0.8-1.8)$ & $7.2(6.1-8.3)$ & $2.9(2.1-3.7)$ & $6.5(5.4-7.6)$ \\
\hline \multicolumn{9}{|l|}{ Disability ${ }^{\dagger+t \dagger}$} \\
\hline Yes & $26.9(24.9-28.9)$ & $23.1(21.2-25.0)$ & $21.1(19.3-22.9)$ & $3.7(2.8-4.6)$ & $1.4(0.9-1.9)$ & $4.2(3.3-5.1)$ & $2.8(2.1-3.5)$ & $5.0(4.1-5.9)$ \\
\hline No & $20.1(19.5-20.7)$ & $16.1(15.5-16.7)$ & $13.3(12.8-13.8)$ & $3.6(3.3-3.9)$ & $0.9(0.8-1.0)$ & $4.5(4.2-4.8)$ & $2.4(2.1-2.7)$ & $3.8(3.5-4.1)$ \\
\hline
\end{tabular}

See table footnotes on the next page. 
Morbidity and Mortality Weekly Report

TABLE. (Continued) Percentage of adults aged $\geq 18$ years who reported tobacco product use "every day" or "some days," by tobacco product and selected characteristics - National Health Interview Survey, United States, 2019

\begin{tabular}{|c|c|c|c|c|c|c|c|c|}
\hline \multirow[b]{2}{*}{ Characteristic } & \multicolumn{8}{|c|}{$\%(95 \% \mathrm{Cl})$} \\
\hline & $\begin{array}{l}\text { Any tobacco } \\
\text { product* }\end{array}$ & $\begin{array}{l}\text { Any combustible } \\
\text { product }^{\dagger}\end{array}$ & Cigarettes $^{\S}$ & $\begin{array}{c}\text { Cigars/Cigarillos/ } \\
\text { Filtered } \\
\text { little cigars }\end{array}$ & $\begin{array}{l}\text { Regular pipe/ } \\
\text { Water pipe/ } \\
\text { Hookah** }\end{array}$ & $\mathrm{E}$-cigarettes ${ }^{\dagger \dagger}$ & $\begin{array}{l}\text { Smokeless } \\
\text { tobacco }^{\S \S}\end{array}$ & $\begin{array}{l}\geq 2 \text { Tobacco } \\
\text { products }\end{array}$ \\
\hline \multicolumn{9}{|c|}{ Generalized anxiety disorder $§ \S \S \S$} \\
\hline None/Minimal & $18.4(17.8-19.0)$ & $14.7(14.1-15.3)$ & $12.0(11.5-12.5)$ & $3.4(3.1-3.7)$ & $0.8(0.7-0.9)$ & $3.6(3.3-3.9)$ & $2.4(2.1-2.7)$ & $3.2(2.9-3.5)$ \\
\hline Mild & $30.4(28.3-32.5)$ & $24.3(22.3-26.3)$ & $21.5(19.5-23.5)$ & $4.0(3.0-5.0)$ & $1.6(1.1-2.1)$ & $8.9(7.5-10.3)$ & $2.3(1.6-3.0)$ & $6.6(5.4-7.8)$ \\
\hline Moderate & $34.2(30.7-37.7)$ & $29.2(25.9-32.5)$ & $27.0(23.8-30.2)$ & $3.9(2.4-5.4)$ & $2.6(1.2-4.0)$ & $9.6(7.3-11.9)$ & $2.2(1.1-3.3)$ & $8.2(6.1-10.3)$ \\
\hline Severe & $45.3(41.1-49.5)$ & $38.7(34.5-42.9)$ & $34.5(30.5-38.5)$ & $6.7(4.5-8.9)$ & $2.1(1.0-3.2)$ & $10.1(7.5-12.7)$ & $3.5(1.9-5.1)$ & $9.4(6.9-11.9)$ \\
\hline
\end{tabular}

Abbreviation: $\mathrm{Cl}=$ confidence interval

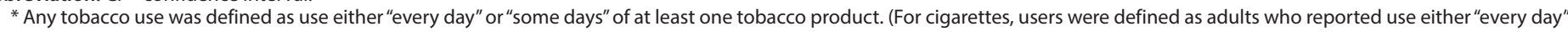
or "some days" and had smoked $\geq 100$ cigarettes during their lifetime).

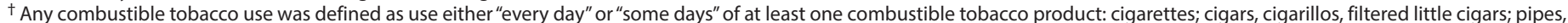
water pipes, or hookah. (For cigarettes, users were defined as adults who reported use either "every day" or "some days" and had smoked $\geq 100$ times during their lifetime).

$\S$ Current cigarette smokers were defined as adults who reported smoking $\geq 100$ cigarettes during their lifetime and now smoked cigarettes "every day" or "some days."

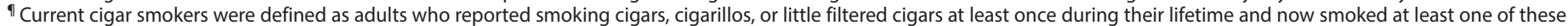
products "every day" or "some days."

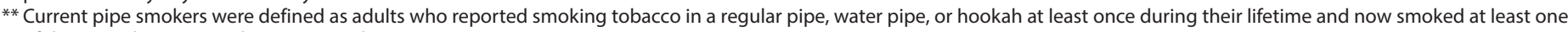
of these products "every day" or "some days."

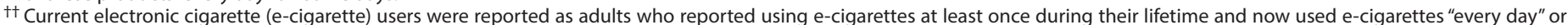
"some days."

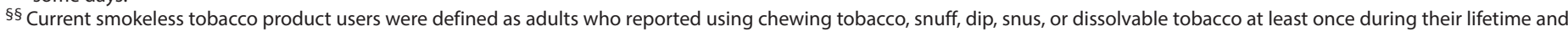
now used at least one of these products "every day" or "some days."

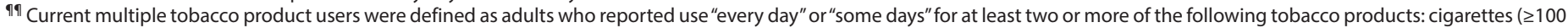
cigarettes during lifetime); cigars, cigarillos, filtered little cigars; pipes, water pipes, or hookah; e-cigarettes; or smokeless tobacco products.

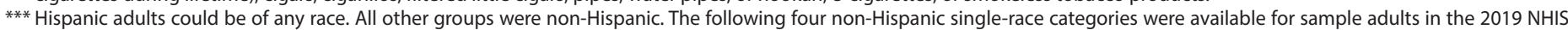

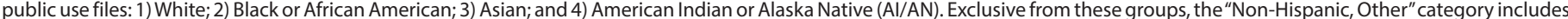

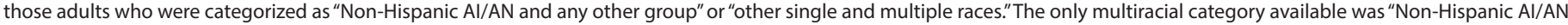
and any other group." ftp://ftp.cdc.gov/pub/Health_Statistics/NCHS/Dataset_Documentation/NHIS/2019/srvydesc-508.pdf.

${ }^{\dagger+\dagger}$ Estimates with a relative standard error $>30 \%$ or unweighted denominator $<50$ are suppressed.

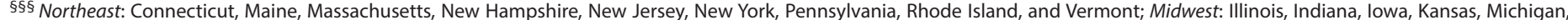

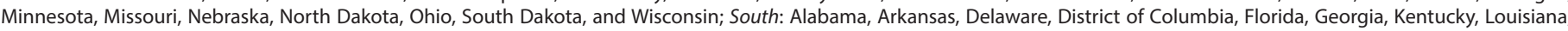

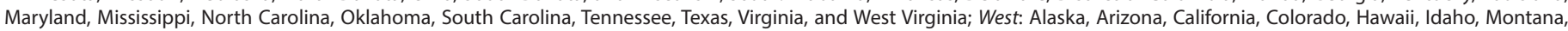
Nevada, New Mexico, Oregon, Utah, Washington, and Wyoming.

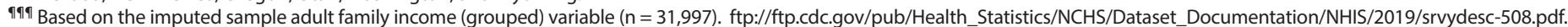

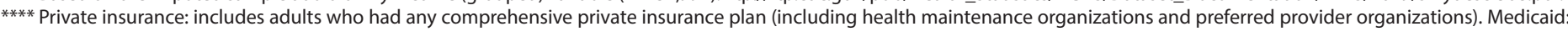

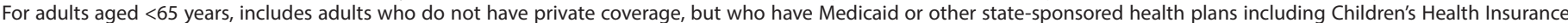

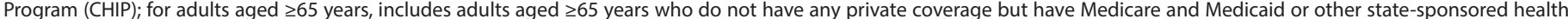

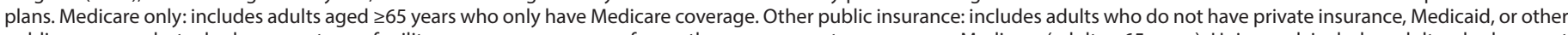

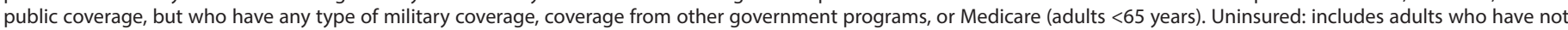

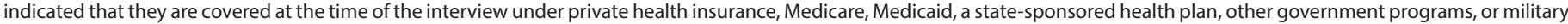
coverage. Insurance coverage is "as of time of survey."

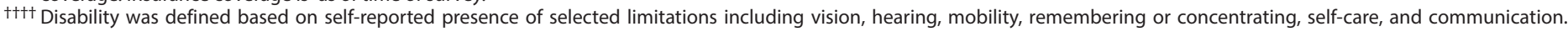

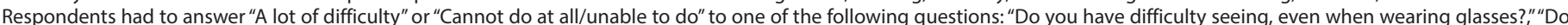

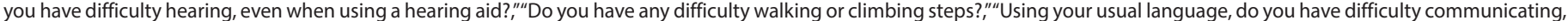

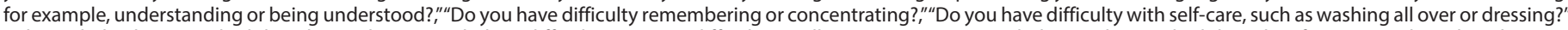

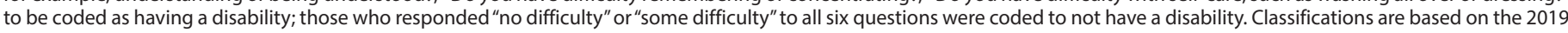

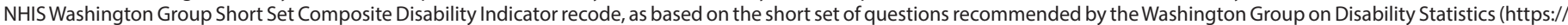
www.cdc.gov/nchs/washington_group/index.htm)

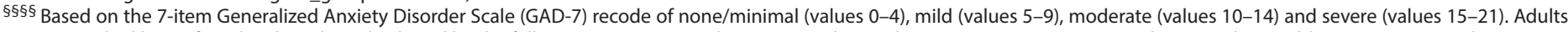

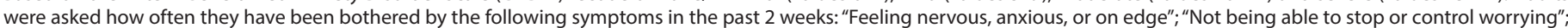

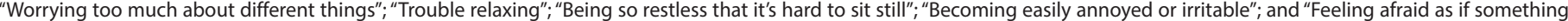

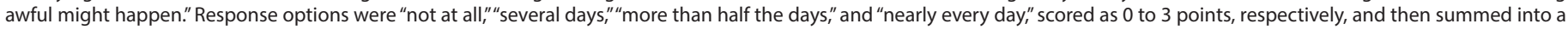
total score.

primarily caused by cigarettes and other combustible products (1); therefore, continued efforts to reduce all forms of combustible tobacco smoking among U.S. adults are warranted. Moreover, approximately one in five current tobacco product users $(18.6 \%)$ reported using two or more tobacco products, and differences in prevalence of tobacco use were also seen across population groups, with higher prevalence among those with a GED, American Indian/Alaska Natives, uninsured adults and adults with Medicaid, and LGB adults. Each of these groups has experienced social, economic, and environmental stressors that might contribute to higher tobacco use prevalence (G). Comprehensive strategies at the national, state, and local levels, including targeted interventions and tailored community engagement, can reduce tobacco-related disease and death and help to mitigate tobacco-related disparities $(1,4,6)$.

U.S. adults also reported using various noncigarette tobacco products, with e-cigarettes being the most commonly used noncigarette tobacco product $(4.5 \%)$. E-cigarette use was highest among adults aged $18-24$ years $(9.3 \%)$, with over half $(56.0 \%)$ of these young adults reporting that they had never smoked cigarettes. In addition, the tobacco product with the highest percentage of users aged 18-24 years $(24.5 \%)$ was e-cigarettes. E-cigarettes contain nicotine, which is highly addictive, can prime the brain for addiction to other drugs, and can harm brain development, which continues until about age 25 years (3). Although e-cigarette use was lower among 
FIGURE 1. Age distribution of adults aged $\geq 18$ years who reported current tobacco product use* - National Health Interview Survey, United States, 2019

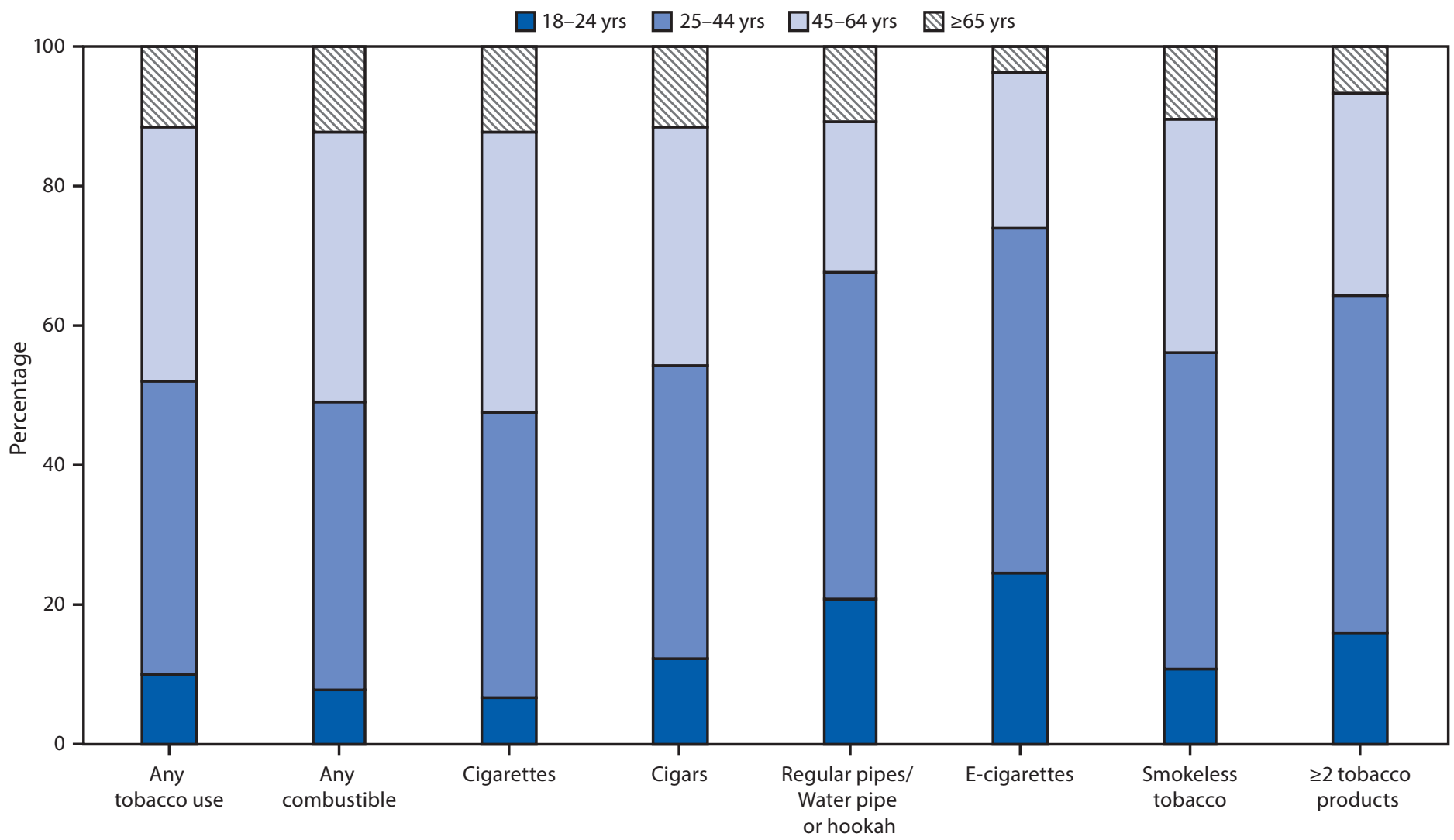

Tobacco product type

\begin{abstract}
* Any tobacco use was defined as use either "every day" or "some days" of at least one tobacco product among individuals. For cigarettes, users were defined as adults who reported smoking $\geq 100$ cigarettes during their lifetime, and smoked "every day" or "some days" at the time of interview. Any combustible tobacco use was defined as use either "every day" or "some days" of at least one combustible tobacco product: cigarettes; cigars, cigarillos, filtered little cigars; pipes, water pipes, or hookah. Use of two or more tobacco products was defined as adults who reported use "every day" or "some days" of at least two or more of the following tobacco products: cigarettes; cigars, cigarillos, filtered little cigars; pipes, water pipes, or hookah; e-cigarettes; or smokeless tobacco products.
\end{abstract}

the older age groups, more than $40 \%$ of e-cigarette users in the $25-44,45-64$ and $\geq 65$ years age groups reported being former smokers. Although some evidence suggests that the use of e-cigarettes containing nicotine and more frequent use of e-cigarettes are associated with increased smoking cessation, smokers need to completely stop smoking cigarettes and stop using any other tobacco product to achieve meaningful health benefits $(6,7)$. The U.S. Surgeon General concluded that there is presently inadequate evidence to conclude that e-cigarettes, in general, increase smoking cessation, and further research is needed on the effects that e-cigarettes have on cessation ( 7 ). Therefore, continued efforts to reduce use of all tobacco products, combustible and noncombustible, are needed.

The findings in this report are subject to at least four limitations. First, the $59.1 \%$ response rate might have resulted in nonresponse bias, although sample weighting is designed to account for this. Second, self-reported responses were not validated by biochemical testing for cotinine (a biomarker indicating nicotine exposure); however, there is high correlation between self-reported smoking and smokeless use and cotinine levels $(8,9)$. Third, because NHIS is limited to the noninstitutionalized U.S. civilian population, these results might not be generalizable to institutionalized populations and persons in the military. Finally, this analysis does not provide comparisons of prevalence estimates with previous surveys because changes in weighting and design methodology for the 2019 NHIS have the potential to affect comparisons of weighted survey estimates over time. $\$ \mathbb{\$ S \$}$

\footnotetext{
\$S\$ 2019 NHIS documentation indicates that changes to the nonresponse adjustment approach and the calibration methods for the 2019 NHIS have the potential to affect comparisons of the weighted survey estimates over time. Because of the changes in weighting and design methodology, direct comparisons between estimates for 2019 and earlier years should be made with caution because the effect of these changes has not been fully evaluated at this time. $\mathrm{ftp}: / / \mathrm{ftp} . c d c . g o v /$ pub/Health_Statistics/NCHS/Dataset_ Documentation/NHIS/2019/srvydesc-508.pdf; https:/www.cdc.gov/ nchs/data/nhis/earlyrelease/EarlyRelease202009-508.pdf.
} 


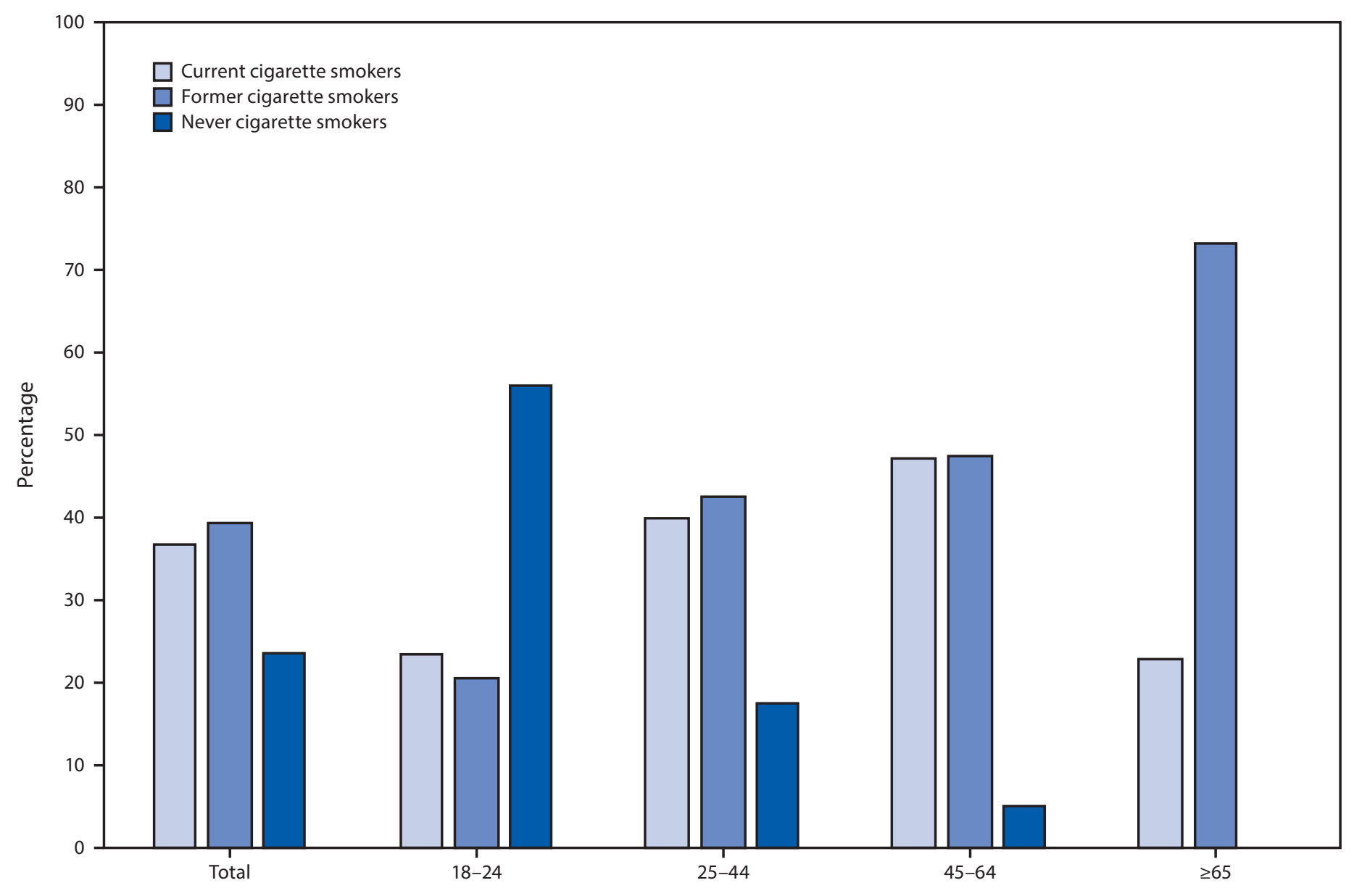

Age group (yrs)

* Adults were asked if they had smoked $\geq 100$ cigarettes in their lifetime and, if yes, whether they currently smoked cigarettes "every day," "some days," or "not at all." Those who smoked "every day" or"some days" were classified as current cigarette smokers. Adults who had not smoked $\geq 100$ cigarettes in their lifetime were classified as never cigarette smokers. Adults who had smoked $\geq 100$ cigarettes in their lifetime but responded to smoking "not at all"at the time of the interview were classified as former cigarette smokers.

† Current e-cigarette users were defined as adults who reported e-cigarette use at least once during their lifetime and use "every day" or "some days" at the time of the interview.

$\S$ The prevalence of never cigarette smokers among e-cigarette users aged 65 years and older is not presented because of relative standard error $>30 \%$ or unweighted denominator $<50$.

The implementation of comprehensive, evidence-based, population-level interventions in coordination with regulation of tobacco products, can reduce tobacco-related disease, disparities, and death in the United States $(1,4)$. These evidencebased, population-level strategies include implementation of tobacco price increases, comprehensive smoke-free policies, high-impact antitobacco media campaigns, and barrier-free cessation coverage (1). As part of a comprehensive approach, targeted interventions are also warranted to reach subpopulations with the highest prevalence of use, which might vary by tobacco product type.

\section{Acknowledgment}

David M. Homa, Office on Smoking and Health, National Center for Chronic Disease Prevention and Health Promotion, CDC.

Corresponding author: Monica E. Cornelius, yex8@cdc.gov, 404-639-3286.

${ }^{1}$ Office on Smoking and Health, National Center for Chronic Disease Prevention and Health Promotion, CDC.

All authors have completed and submitted the International Committee of Medical Journal Editors form for disclosure of potential conflicts of interest. No potential conflicts of interest were disclosed. 


\section{Summary}

What is already known about this topic?

Cigarette smoking remains the leading cause of preventable disease and death in the United States; however, a variety of new combustible, noncombustible, and electronic tobacco products are available in the United States.

What is added by this report?

In 2019 , approximately $20.8 \%$ of U.S. adults (50.6 million) currently used any tobacco product. Cigarettes were the most commonly used tobacco product among adults, and e-cigarettes were the most commonly used noncigarette tobacco product (4.5\%). The highest prevalence of e-cigarette use was among smokers aged $18-24$ years $(9.3 \%)$, with over half (56.0\%) of these young adults reporting that they had never smoked cigarettes.

What are the implications for public health practice?

The implementation of comprehensive, evidence-based, population-level interventions, combined with targeted strategies, in coordination with regulation of tobacco products, can reduce tobacco-related disease and death in the United States. As part of a comprehensive approach, targeted interventions are also warranted to reach subpopulations with the greatest use, which might vary by tobacco product type.

\section{References}

1. US Department of Health and Human Services. The health consequences of smoking — 50 years of progress: a report of the Surgeon General. Atlanta, GA: US Department of Health and Human Services, CDC; 2014. https://www. ncbi.nlm.nih.gov/books/NBK179276/pdf/Bookshelf_NBK179276.pdf
2. Creamer MR, Wang TW, Babb S, et al. Tobacco product use and cessation indicators among adults_-United States, 2018. MMWR Morb Mortal Wkly Rep 2019;68:1013-9. PMID:31725711 https://doi.org/10.15585/mmwr. mm6845a2

3. US Department of Health and Human Services. E-cigarette use among youth and young adults: a report of the Surgeon General. Atlanta, GA: US Department of Health and Human Services, CDC; 2016. https:/e-cigarettes. surgeongeneral.gov/documents/2016_SGR_Full_Report_non-508.pdf

4. CDC. Best practices for comprehensive tobacco control programs-2014. Atlanta, GA: US Department of Health and Human Services, CDC; 2014. https://www.cdc.gov/tobacco/stateandcommunity/best_practices/index. htm?source=govdelivery

5. National Center for Health Statistics. Survey description, National Health Interview Survey, 2019. Hyattsville, MD: US Department of Health and Human Services, CDC, National Center for Health Statistics; 2020. $\mathrm{ftp} / / / \mathrm{ftp} . \mathrm{cdc}$.gov/pub/Health_Statistics/NCHS/Dataset_Documentation/ NHIS/2019/srvydesc-508.pdf

6. National Cancer Institute. A socioecological approach to addressing tobaccorelated health disparities. National Cancer Institute Tobacco Control Monograph 22. NIH publication no. 17-CA-8035A. Bethesda, MD: US Department of Health and Human Services, National Institutes of Health, National Cancer Institute; 2017. https://cancercontrol.cancer.gov/sites/ default/files/2020-08/m22_complete.pdf

7. US Department of Health and Human Services. Smoking cessation. A report of the Surgeon General. Atlanta, GA: US Department of Health and Human Services, CDC; 2020. https://www.hhs.gov/sites/default/files/2020-cessationsgr-full-report.pdf

8. Binnie V, McHugh S, Macpherson L, Borland B, Moir K, Malik K. The validation of self-reported smoking status by analysing cotinine levels in stimulated and unstimulated saliva, serum and urine. Oral Dis 2004;10:287-93. PMID:15315646 https://doi.org/10.1111/j.1601-0825.2004.01018.x

9. Agaku IT, King BA. Validation of self-reported smokeless tobacco use by measurement of serum cotinine concentration among US adults. Am J Epidemiol 2014;180:749-54. PMID:25125690 https://doi.org/10.1093/ aje/kwu182 